\title{
Simple method of computing a function in fixed-point arithmetic
}

\author{
S.S. Deo and K.V.S. Hari
}

Indexing term: Digital arithmetic

The authors present a simple method, based on the principle of successive approximation, of computing a function using a fixedpoint arithmetic processor. As an example, computing the square root function is considered and the performance is compared with Newton's method, in terms of accuracy and the number of instruction cycles required.

Introduction: The method of successive approximation (SA) is most commonly used in analogue to digital converters (ADCs). A simple method, based on this principle, for the computation of functions in fixed-point arithmetic is proposed. The effectiveness of this method in terms of accuracy, number of cycles etc., 
depends on how effectively the inverse function is computed. This Letter presents the algorithm and presents the performance of the algorithm for square root computation on a fixed-point DSP processor.

Successive approximation (SA) method for computing a function: Consider a $B$ bit fixed-point arithmetic processor and assume that the values are represented in fractional format as

$$
X=b_{s} \cdot b_{0} b_{1} \ldots b_{B-2}
$$

with $b_{s}$ denoting the sign bit. Consider the function

$$
\mathrm{Y}=f(x)
$$

which needs to be evaluated and let $f^{1}(\cdot)$ denote the inverse function.

\section{Algorithm:}

$y_{0}<=" b_{s}=0, b_{0}=1, b,=0, i \neq 0 "$; initial guess (usually mid-point)

$y_{1}<=" b_{s}=0, b_{1}=1, b_{1}=0, i \neq 1 " ;$ a constant named shift-Val-1

$y_{2}<=" b_{s}=0, b,=1, b_{i}=0, i \neq 0=1, " ;$ a constant named shift-val-2

FOR (LOOP $=1$ to $B-1)$

START LOOP

IF $\left(x>f^{1}\left(y_{0}\right)\right)$

$y_{0}<=y_{0}$ OR $y_{1}$;

shift right $y_{1}$ by 1 bit position;

shift right $y$, by 1 bit position;

$$
\text { END IF }
$$

$\operatorname{IF}\left(x<f^{1}\left(y_{0}\right)\right)$

$y_{0}<=Y_{0}$ AND (NOT $y_{2}$ ) OR $y_{1}$

shift right $y_{1}$ by 1 bit position;

END IF

shift right $y_{2}$ by 1 bit position;

IF $\left(x=f^{-1}\left(y_{0}\right)\right)$

\section{END IF}

TERMINATE LOOP;

\section{END LOOP}

$f(x)<=y_{0}$

STOP

\section{Remarks:}

(i) The number of iterations required for computation are equal to the number of bits used for representing $y$.

(ii) The accuracy and computational complexity of implementing the inverse function dictates the accuracy and computational complexity of the SA method of function evaluation.

Examples: Square root computation: Usually, the square root of a number is evaluated using the Newton Raphson method given by the iterative formula [1]:

$$
y(n)=\frac{1}{2}\left[y(n-1)+\frac{\|}{y(n-1)}\right]
$$

where $n$ denotes the $n$th iteration. After some iterations, $y(n)$ approximates to $\sqrt{x}$.

Using the SA method, it is easy to see that for square root computation, the inverse function is simply squaring, or multiplication. This can effectively be carried out by a hardware multiplier in one clock cycle, which is part of a digital signal processing (DSP) processor (for e.g. [4]).

Performance comparison: A uniform random number generator was used to generate 100 random numbers between zero and one, and the square root was calculated for each of them using the two methods. The average error was chosen as the performance measure. Table 1 presents the results.

\begin{tabular}{|c|c|c|c|}
\hline Method & Cycles per iteration & Iterations & Average error \\
\hline Newton-Raphson & 32 & 40 & $5 \times 10^{-4}$ \\
\hline Successive approx & 24 & 15 & $2 \times 10^{-5}$ \\
\hline
\end{tabular}

Sine inverse computation: The proposed method was also used for computing the arcsin function in [5] and it has been shown that it performs better than the standard method using a series expansion [2].

Conclusion: A simple method, the SA method, is proposed for computing a function in fixed-point arithmetic. The method is general and its power lies in efficient implementation of the inverse function. As an example, it has been shown that using a DSP processor, computing the square root using the proposed method is more accurate and requires less computation compared to the conventional Newton-Raphson method.

\section{References}

1 PROAKIS, J.G., and MANOLAKIS, D.G.: 'Digital signal processing principles, algorithms and applications' (Prentice Hall of India, 1995)

2 DSP Applications using ADSP 2100 Family. Analog Devices, 1992

3 ADSP 210112102 Ez-Lab Manual. Analog Devices, 1990

4 ADSP 2100 Family User's Manual. Analog Devices, 1993

5 DEO, S.S.: 'Digital signal processing based interface for a fiber optic gyroscope'. M.E. Thesis, Department of ECE, Indian Institute of Science, Bangalore, India, 1997 